\title{
Overtraining Syndrome
}

\author{
R. Budgett, MBBS, MA, Dip Sports Med, MRCGP \\ The British Olympic Medical Centre
}

\begin{abstract}
This review discusses the overtraining syndrome which is characterized by fatigue and underperformance precipitated by stress of training. Other stresses, depression and an increased susceptibility to infections may be important. Treatment requires rest and a stress management program over 3 months.
\end{abstract}

Keywords: Stress, underperformance, overtraining syndrome, staleness, chronic fatigue

\section{Introduction}

There have been numerous anecdotal reports of athletes underperforming for no apparent reason and taking weeks to recover form. The term 'overtraining syndrome' is often used to describe these athletes suffering from prolonged fatigue. This term implies causation, but there is no firm evidence for this, and athletes who complain of fatigue and underperformance may not all be suffering from a single pathophysiological entity.

Other terms such as 'burnout', 'staleness', and 'chronic fatigue syndrome' have been used to describe a similar picture. Some commentators feel the problem is post-viral in origin linking it with $\mathrm{ME}^{1}$. The picture is complicated by a growing number of reports of immune dysfunction after prolonged exhaustive training, so that frequent or prolonged infections causing fatigue and malaise may be secondary to overtraining rather than the primary cause of the problem.

\section{Definitions}

\section{Fatigue}

This is a subjective condition synonymous with tiredness, lethargy and listlessness, with symptoms such as poor concentration and poor tolerance of activity. It contrasts with physiological fatigue which can be defined as the failure to sustain an expected or required muscular force. The mechanism depends on the duration of exercise, so that a sprinter will become fatigued in seconds in association with high

Address for correspondence: Dr R. Budgett, The British Olympic Centre, Northwick Park Hospital, Watford road, Harrow, Middlesex HA1 3UJ, UK

(C) 1990 Butterworth-Heinemann Ltd

0306-3674/90/040231-06 lactate levels, while a marathon runner will fatigue after nearly 2 hours due to glycogen depletion ${ }^{3}$. Fatigue in this review refers to the subjective condition.

\section{Overtraining syndrome}

1. This may be seen as prolonged fatigue and underperformance, following a period of heavy training or competition, lasting at least two weeks and confirmed on an appropriate ergometer, or by comparing training times or results in previous competition. Identifiable medical illness must have been excluded. This assumes that the stress of training is the major factor in the aetiology of prolonged fatigue in athletes.

2. Athletes who suffer recurrent infections during periods of maximum training or competition stress may suffer from overtraining syndrome.

This assumes that overtraining is the major stress causing immune dysfunction, producing increased vulnerability to infection.

\section{Burnout/staleness}

The definition is as for 1 . in the overtraining syndrome. It is often used to describe swimmers ${ }^{4-5}$.

\section{Chronic fatigue syndrome}

This is the same definition as 1 . in the overtraining syndrome. The term is used in American literature ${ }^{6, ?}$.

\section{Post-viral infection/ME}

This causes fatigue lasting many months following a viral, possibly enteroviral, infection ${ }^{8}$. Patients are unable to function in normal life let alone train. Few athletes fall into this category. There is no evidence that the proposed reduction in immune function in overtrained athletes predisposes to post-viral fatigue, so this cannot yet be defined as part of the overtraining syndrome.

\section{Sympathetic and parasympathetic staleness}

These terms have been used to sub-divide the overtraining syndrome, but are not generally used now due to the lack of evidence for a separate parasympathetic type which was supposedly characterized by hypoglycaemia during exercise and 
exhaustion of the neuro-endocrine system. The sympathetic type was defined as essentially a response to prolonged stress and fits with overtraining definition $1 .^{9-1}$.

\section{Over-reaching}

Purposeful overtraining may be part of a planned programme in order to stimulate adaptation. This is followed by a period of relative rest or tapering in order to allow full supercompensation, normally within 1-2 weeks ${ }^{12}$.

\section{Clinical picture}

As suggested by the two definitions, there are two broad clinical presentations which may overlap.

Athletes may suffer from fatigue and a performance slump which does not improve despite resting over 2 days or reducing the training load for 2 weeks. They are often highly motivated, conscientious individuals who have responded to their lack of form by increasing the training load, leading to a vicious cycle of fatigue and underperformance ${ }^{12}$.

Others have been entering a series of hard competitions, often with initial success, but then begin to become tired and produce steadily worse results ${ }^{13}$. These athletes are characterized by their reluctance to stop or reduce their level of training and competition due to their own ambition and determination sometimes combined with pressure from the coach, fellow athletes and parents.

Other elite competitors build up their training for two to three months, reaching a high level of performance, but then suffer from a viral infection which stops them in their tracks. This often occurs around the time of a major competition or series of races. After enforced rest for 1-2 weeks they restart training, but when they reach a certain level again break down and the cycle repeats itself ${ }^{14,15}$. As yet, there is only indirect evidence of immune dysfunction (Riddell: personal communication).

Reports of athletes presenting in this way are nearly all anecdotal, but the constant flow of reports from coaches and athletes show that they perceive there is a problem despite the fact that it is poorly defined ${ }^{12}$. A large number of symptoms and signs have been described, but some of them relate to athletes who are only over-reaching, and others may represent underlying unidentified disease since they are inconsistently reported in different studies. There is no accepted gold standard for the diagnosis of the so called overtraining syndrome.

\section{Symptoms}

Athletes can only be labelled as overtrained if they are fatigued and underperforming. They may complain of fatigue at rest, with a loss of purpose, energy and competitive drive ${ }^{16,17}$. Feelings of helplessness, incompetence, being trapped in a routine, and emotional lability and loss of libido have been described $^{10,13}$. Athletes often admit to increased anxiety and depression (90\%), irritability (70\%) and sleep problems $(90 \%)$ including difficulty getting to sleep, nightmares and waking in the night ${ }^{18}$. Loss of appetite, weight loss, heavy painful muscles and excessive sweating have been reported ${ }^{4,11,13}$. Athletes have also been noted to complain of an increased susceptibility to infections ${ }^{2,16}$ (Riddell: personal communication) and injuries ${ }^{19}$.

\section{Signs}

There are no physical signs consistently associated with the overtraining syndrome. The following have been reported in association: raised early morning heart rate, increased postural drop in blood pressure and rise in heart rate, and a slow return of pulse rate to normal after exercise ${ }^{20,21}$, decreased maximal lactate levels during exercise and decreased maximal power output ${ }^{10}$. There may be lymphadenopathy, diarrhoea, increased susceptibility to allergies and minor scratches may heal slowly ${ }^{13}$ (Riddell: personal communication). However, none of these signs are reliable indicators of the syndrome, since they can appear during periods of over-reaching without any drop in performance. They are reported inconsistently and some may represent underlying disease.

\section{Precipitating factors}

\section{Physical stress}

Stresses other than those of hard training and competition may lead to an inability to recover from a previously well tolerated training programme.

Glycogen depletion leads to rapid fatigue particularly after the anaerobic threshold is reached, and may predispose to more prolonged fatigue ${ }^{22}$. Thus, a poor diet with insufficient carbohydrates to meet training demands ${ }^{23}$, as may occur when an athlete is attempting to lose weight, will increase the difficulty in coping with a particular programme.

Dehydration will also increase the perceived stress of exercise and is a potent cause of underperformance $^{24}$.

Infections, as well as being reported more frequently in hard training, will make it more difficult for athletes to tolerate the load.

\section{Psychological stress}

The profile of mood states questionnaire asking athletes to rate their current feelings or mood against a list of 65 words has been used to follow them in training. It shows reduced vigour and increased tension, depression, anger, fatigue and confusion (inverted iceberg profile) during periods of heavy training ${ }^{25,26}$. This is even worse in those athletes who suffer from prolonged fatigue and are labelled as stale or overtrained ${ }^{27,28}$. It has been suggested that academic, economic and social stressors may be important. They certainly feature prominently in anecdotal reports. However, there is no good evidence for their role due to the lack of studies on competitors who become overtrained. 


\section{Differential diagnosis}

\section{Serious disease}

This is fortunately rare but it is important to exclude viral myocarditis or arrhythmias, bearing in mind that electrocardiograms are often abnormal, particularly at rest, in highly trained athletes ${ }^{29}$.

\section{Glycogen depletion}

Prolonged inadequate carbohydrate intake may cause prolonged fatigue as well as precipitating a true overtraining syndrome.

\section{Anaemia}

It is important to distinguish between true anaemia which may be due to iron deficiency, particularly in female athletes ${ }^{30}$, and the physiological reduction in haemoglobin due to increased plasma volume which has no effect on performance. Low iron stores as measured by serum ferritin levels were thought to be responsible for many cases of underperformance ${ }^{31}$, but it has been shown that levels as low as $12 \mathrm{ng} / \mathrm{ml}$ are not associated with underperformance. There is no advantage in raising this with iron supplements ${ }^{32}$.

\section{Exercise induced asthma}

This diagnosis is easily missed unless enquired for directly, and is a common cause of apparent recurrent colds and underperformance ${ }^{33}$. A $15 \%$ or greater fall in peak expiratory flow rate or FEV1 after exercise is considered diagnostic ${ }^{34}$.

\section{Physical changes due to age}

These are sometimes difficult to accept! The change in shape of female athletes, such as gymnasts or swimmers, at menarche is particularly dramatic if they have a few months off and put on weight. This will greatly affect performance but should not cause fatigue ( $P$. Garret: personal communication).

\section{Psychological}

There is a psychological element to every athlete's underperformance, but in some this is of overriding importance so that the training stress is irrelevant. In these cases the athlete cannot be labelled as overtrained, and psychological help is far more important than rest from training.

\section{Prevention}

It is obviously important to avoid the precipitating factors discussed earlier, but early detection and a carefully designed training programme may also play a role in prevention.

\section{Early detection}

The careful monitoring of athletes who are overreaching has been suggested in order to pick up true overtraining or staleness in the early stages ${ }^{21}$. In general, the longer the history of fatigue before training is stopped, the longer recovery takes ${ }^{12}$. The drop in performance often become obvious too late to avoid a prolonged period of fatigue. Because of this, early morning heart rate ${ }^{20}$ and mood state questionnaires ${ }^{27}$ have been used. Others have suggested using serial measurements of haemoglobin, haematocrit or creatine kinase. Unfortunately, these tests are non-specific and reflect training load or may not even change at all ${ }^{10,16}$. Thus, there is no objective early screen that can be reliably used to detect those athletes who will become overtrained during a period of overreaching.

\section{Training}

There is consensus that undertraining is better than overtraining, and this seems to be the surest way of avoiding prolonged fatigue ${ }^{21}$. However, maximal training is needed for an athlete to reach their full potential and various strategies have been suggested to allow this while minimizing the risk of the overtraining syndrome. Sudden increases in training load should be avoided, a steady increase of $5 \%$ per week being optimal for most athletes. Intensive exercise with short rests and frequent competition is dangerous. Monotonous training should also be avoided, particularly in swimmers ${ }^{11}$. Notice should be taken of other stresses, both physical and psychological, since these may combine and exceed an individual's ability to adapt. Training should be reduced at these times?.

\section{Management \\ Rest}

Cases need to be assessed individually, and it is often difficult to persuade athletes that they need to rest. However, this seems to be the basis of treatment since an improvement in performance has been shown after 3-5 weeks rest, despite the fact that these athletes must become detrained in this time ${ }^{28}$. The build up to full training may take up to three further months. It is thought important to avoid competition $^{13}$, although individuals may produce personal best performances 'out of the blue' before apparent complete recovery ${ }^{6}$.

In the majority of athletes who are still able to work and function in normal life, a regime of relative rest has been suggested using gentle non-competitive exercise often in a different sport with no measure of performance available. Usually this low level of exercise is easily tolerated and the psychological and physical benefits will help speed recovery ${ }^{4}$, while maintaining a degree of cardiovascular fitness. The emphasis should be on exercise as a therapeutic tool to speed rehabilitation, rather than as a means to obtain a training effect which is what the athletes are used to.

\section{Regeneration}

No controlled trials of treatment have been reported, but regeneration strategies have been widely used 
particularly in the Eastern bloc ${ }^{35}$. This involves a reduction in all stresses with rest, sleep, relaxation therapy and counselling as well as saunas, massage, hydrotherapy and physiotherapy $y^{5}$. Nutrition is looked at in detail and vitamins and other supplements given routinely although there is no proven benefit. Psychotherapy and counselling have been used in the USA to successfully treat underperforming athletes diagnosed as stale and depressed ${ }^{25}$, but there are no reports on the use of antidepressants. However, there is one report of anabolic steroid use in an attempt to speed recovery ${ }^{36}$, and no doubt others have tried this unproven and illegal tactic without reporting their results. Our own anecdotal observation suggests that recovery takes three to eight weeks. However, there is a danger of relapse at around 3 months. We advise reduced training and competition stress for up to 4 months.

\section{Investigating the overtraining syndrome}

There are only a few studies of truly overtrained elite competitors ${ }^{18,25,27,28,37}$ (Riddell: personal communication). Most investigators have concentrated on studying the response to severe exhaustive exercise in an attempt to find markers of and mechanisms to explain the overtraining syndrome. This ignores the previously discussed problem that overtrained competitors may be training no harder than their peers, and that it is something unique to that individual that causes prolonged breakdown with failure to adapt or benefit from training. Moreover, in those studies in which performance is monitored, there is generally a quick recovery or even an improvement at the end of the period of extreme over-reaching ${ }^{38}$.

\section{Routine tests as markers}

Haemoglobin and haematocrit have been shown to fall during periods of heavy training ${ }^{39}$, but this is probably physiological, and does not correlate with performance. Others have found no change ${ }^{16}$. ECGs are often abnormal in fit athletes and are no different during periods of heavy training or underperformance $^{29}$.

Creatine kinase has been used by many researchers, but unfortunately has been shown to rise in proportion to training load, presumably due to muscle damage, does not correlate with performance, and there is wide individual variability $(50 \mathrm{fold})^{13,20}$. In a single study, thyroxine and TSH were reduced during a period of prolonged physical and mental stress, but levels did not correlate with performance and recovery took only two days.

\section{Hormonal and endocrine changes}

Cortisol is catabolic, helping to mobilize glycogen and free fatty acids, and rises after hard exercise as part of the stress hormone response ${ }^{10,40,41}$. Psychological stress also raises levels, particularly before competition $^{42}$. Testosterone has also been shown to rise in response to exericse, and there is evidence that levels are higher in well trained individuals ${ }^{17,43,44}$.
Intense and prolonged training will raise cortisol levels even higher, but will reduce testosterone $e^{45-47}$. Thus, it has been suggested that hormonal changes are involved in or at least are markers of the overtraining syndrome ${ }^{17}$. It may be that at a critical testosterone/cortisol ratio the predominance of catabolism over anabolism means that an athlete is unable to recover from training, and continues to break down rather than adapting positively.

Harkonnen et al. looked at 15 long distance runners, and increased the training load of nine of them for just one week $^{48}$. The testosterone/cortisol ratio fell in eight of the nine who were classified as overtrained and in only one control. There was no record of chronic fatigue or underperformance. These athletes were therefore not truly overtrained, but merely exhibited a normal response to extreme training stress.

$\mathrm{O}^{\prime}$ Connor et al. followed salivary cortisol levels in 14 female swimmers over 6 months ${ }^{27}$. As well as the expected rise in baseline levels as training increased from 2,000 to 12,000 yards per day, they found significantly higher levels in those suffering from staleness and underperformance compared to those maintaining their performance. This correlated with depressed mood state ${ }^{27}$.

Barron and Noakes in 1985 suggested that the overtraining syndrome was due to hypothalamicpituitary dysfunction ${ }^{37}$, as may be the case in female athletes who develop amenorrhoea. They found a significantly reduced hormonal response to insulin induced hypoglycaemia in four chronically fatigued and underperforming athletes compared to controls. However, basal cortisol levels were raised, making the interpretation of the results difficult, but further supporting the existence of high basal cortisol levels in overtrained athletes. Testosterone was not measured.

\section{Mood state}

Some commentators feel that the chronic fatigue seen in athletes is 'depression with a new face ${ }^{7 \prime}$. It is possible that the stress of heavy training and competition, combined with other stresses, can precipitate depression. This would certainly explain why some athletes are more susceptable to the so called overtraining syndrome than others. There is no doubt that the athletes are depressed with changes in global mood as measured by the Profile of Mood States (POMS) questionnaire $27,28$.

The symptoms of overtraining are very similar to those described in depression with psychomotor and physiological retardation, chronic fatigue, depressed appetite, weight loss, insomnia, muscle soreness, and tension and depression. However, the mood state changes in response to an increased training load are non-specific, with a decrease in vigour and increase in tension, depression, anger, vigour and confusion in all athletes, so it is difficult to use a mood state questionnaire to predict or diagnose the overtraining syndrome. Despite this, there is evidence that this change is even greater in underperforming athletes ${ }^{27}$ and so may potentially be used to 
support the diagnosis and monitor recovery since mood state improves with rest in parallel with performance $^{28}$.

\section{Immune function}

Anecdotal reports have linked susceptibility to infections to periods of heavy training, sometimes associated with prolonged fatigue and underperformance $^{14,15}$ (Riddell: personal communication).

Many studies have investigated the effect of exercise on the immune system, and it is now accepted that there is a release of white blood cells causing a temporary leucocytosis after heavy exercise $^{2}$. However, there is no evidence that this of functional significance.

Some studies have shown a reduced $\mathrm{T}$ helper: $\mathrm{T}$ suppressor cell ratio after hard exercise ${ }^{49,50}$, but this did not correlate with temporary suppression of lymphocyte function which has also been described $^{51}$. Makinnon showed reduced salivary $\operatorname{IgA}$ and IgM concentrations and reduced NK cell activity after one to two hours of heavy exercise ${ }^{52}$. However, these changes resolved soon after exercise, and are not found consistently. Nash showed that training enhanced immune function ${ }^{53}$. Thus, there is still uncertainty about the effect of exercise and training on immune function which may depend on the pre-existing state of the athlete, and the type of exercise.

It is difficult to isolate the effect of psychological stress which has been shown to be capable of suppressing immunity ${ }^{2}$ maybe by raising cortisol levels ${ }^{54}$, although immunosuppression may occur in adrenalectomized animals ${ }^{55}$.

Another explanation for the widely reported, but as yet unproven, susceptibility of athletes to infections during periods of chronic fatigue and underperformance, is through the reduced plasma glutamine levels which have been found in overtrained athletes ${ }^{18}$. This amino-acid is produced in muscle from the breakdown of other amino-acids $s^{56}$, and is an essential fuel for all rapidly dividing cells ${ }^{57}$. It donates nitrogen for purine synthesis, as well as being oxidized to provide energy. It also provides $35 \%$ of the energy for lymphocyte metabolism ${ }^{58}$. Thus, low plasma levels may cause immunosuppression, leading to frequent infections and a worsening cycle of underperformance.

\section{Virology}

It has been suggested that the majority of cases of chronic fatigue in athletes are post-viral in origin ${ }^{1}$. Infectious mononucleosus is a well known cause of chronic fatigue, and there have been cases of proven infection with enteroviruses and coxsackie $B^{18}$, but the relevance of high titres to underperformance has been questioned. Subclinical infection or high titres did not correlate with objective or subjective underperformance in a group of 698 athletes ${ }^{15}$. In another study of 12 fatigued athletes, there were two positive monospots with atypical mononuclear cells (glandular fever), and two raised coxsackie B titres. However, no cause was found in the other eight ${ }^{59}$.
If a definite viral cause is found, then a diagnosis of the overtraining syndrome cannot be made unless it is part of a pattern of recurrent infections at times of heavy training and competition stress. However, high viral titres have to be interpreted in their clinical context, and may be an incidental finding in fatigued competitors.

\section{Conclusion}

Current evidence on the pathophysiology of the overtraining syndrome is limited, but there is no doubt that the problem of unexplained underperformance does exist.

Investigators have suggested hormonal, psychological, immunological and post-viral aetiologies, and it is possible that all these play a part in different athletes. Increased catabolism, depression, infections, and the stress of training combined with other stresses, may all lead to fatigue and underperformance. The stress of training can cause depression, raised cortisol levels and reduced plasma glutamine levels which may all contribute to decreased immune function.

Management consists of rest and stress reduction through a regeneration programme including counselling and relaxation therapy. Recovery takes three to eight weeks.

\section{References}

1 Kevles, B. Flat tired out: How EBV, the mysterious chronic fatigue syndrome, has brought a group of women cyclists to a grinding halt City Sports Magazine 1988, 14 (9), 17-22

2 Keast, D. Exercise and the immune response Sports Medicine 1988, 5, 248-267

3 Brooks, G.A. and Fahey, T.D. Fatigue during muscular exercise Exercise Physiology Macmillan Publishers, London, 1984

4 Burke E. Blood testing to determine overtraining in swimmers Swimming technique 1981, 18 Nov; 29-33

5 Crampton, J. Regeneration vs burnout: prevention is better than cure! Sports Coach 1987, April/June, 7-11

6 Eichner, E. Chronic fatigue syndrome: How vulnerable are athletes? Phys Sportsmed 1989, 17 (6), 157-160

7 Eichner, E. Chronic fatigue syndrome: Searching for the cause and treatment Phys Sportsmed 1989, 17 (6), 142-152

8 David, A.S. Postviral fatigue syndrome: Time for a new approach Br Med J (Clin Res) 1988, 296, 696-699

9 Israel, S. Zur Problematic des Uebertrainings aus internistischer und leistungsphysiologischer Sicht Medizin und Sport 1976, 16 (1), 1-12

10 Kinderman, W. Das Ubertraining-Ausdruck einer vegitativen Fehlsteuerung Deutsche Zeitschrift fur Sportmedizin 1986, H8, 138-145

11 Kuipers, H. and Keizer, H.A. Overtraining in elite athletes Sports Medicine 1988, 6, 79-92

12 Brasher, C. The under-achievers who overtrain Observer: January 17th 1988

13 Noakes, T. Lore of Running Oxford University Press, Cape Town, 1986

14 Peters, E.M. Ultramarathon running and upper respiratory tract infections: An epidemiological survey South African Med J 1983, 64, 582-584

15 Roberts, J.A. Virus illness and sports performance Sports Med 1986, 3, 298-303

16 Costill, D.L. Inside Running Benchmark Press Inc. Indianapolis, 1986 
17 Adlercreutz, $\mathrm{H}$. Effect of training on plasma anabolic and catabolic steroid hormones and their response during physical exercise Int J Sports Med 1986, 7, 27-28 (supplement)

18 Budgett, $R$. et al. The overtraining syndrome/staleness (Abstract) International Olympic Committee World Congress on Sports Sciences, Colorado Springs, 1989

19 Barron, D. K. and Mellerowicz, H. Overtraining Encyclopedia of Sports Sciences and Medicine (Eds Larson and Leonard), Macmillan, New York, 1971

20 Dressendorfer, R.H. Increased morning heart rate in runners: a valid sign of overtraining? Phys Sportmed 1985, 13 (8), 77-86

21 Ryan, A. Overtraining in athletes: A round table The Physician and Sports Medicine 1983, 11 (6), 93-110

22 Costill, D.L. Effects of repeated days of intensified training on muscle glycogen and swimming performance Med Sci Sports Exerc 1988, 20 (3), 249-54

23 Wheeler, K. Sports nutrition for the primary care physician: The importance of carbohydrate Phys Sportsmed 1989, 17 (5), 106-117

24 Costill, D.L. Nutrition and dietetics Olympic Book of Sports Medicine (Eds Dirix, A., Knuttgen, H. and Tittel, K.), Blackwell Scientific Publications, Oxford, 1988

25 Morgan, W.P. Psychological monitoring of overtraining and staleness Brit J Sports Med 1987, 21 (3), 107-114

26 Costill, D.L. and Morgan, W.P. Mood disturbance following increased training in swimmers Med Sci Sports Exerc 1988, 20 (4), 408-414

27 O'Connor, P.J. et al. Mood state and salivary cortisol levels following overtraining in female swimmers Psychoneuroendocrinology 1989, 14 (4), 303-310

28 Koutedakis, Y., Budgett, R. and Fullman, L. The role of physical rest for underperforming elite competitors $\mathrm{Br} J$ Sports Med (in press)

29 Hollman, W. Sportsmedizin, Arbeits und Trainingsgrundlagen, F.K. Schattauer Verlag, Stuttgart, 1980

30 Haymes, E.M. Nutrition for the female distance runner (ed) Drinkwater, B.L. Female Endurance Athletes Human Kinetics Publishers, Champaign, 1986

31 Eichner, E. The anaemias of athletes. Phys Sports Med 1986, 14 (9), 122-130

32 Newhouse, I., Clement, D., Taunton, J. and McKenzie, D. The effects of prelatent/latent iron deficiency on physical work capacity. Med Sci Sports Exerc 1989, 21 (3), 263-268

33 McCarthy, P. Wheezing or breezing through exercise-induced asthma Phys Sportsmed 1989, 17 (7), 125-130

34 Szabadi, E. Physical exercise and mental health $\mathrm{Br}$ Med J 1988, 296, 259-260

35 Paikov, V.B. Means of restoration in the training of speed skaters (condensed) Soviet Sports Review 1985, 20 (1), 9-12 (Translated from Konkobezhniv Sport 1982, 1, 39-43)

36 Kereszty, A. Overtraining Encyclopedia of Sports Sciences and Medicine (ed) Larson and Leonard, 218-222, MacMillan, New York, 1971

37 Barron, G.L. and Noakes, T.D. Hypothalamic dysfunction in overtrained athletes J Clin Endocrin Metab 1985, 60 (4), 803-806

38 Costill, D.L. Physiological response to successive days of intense training in competitive swimmers Med Sci Sports Exerc 1988, 20 (3), 255-259
39 Rushall, B.S. Haematological responses to training elite swimmers Canadian Journal of Applied Sports Sciences 1980, 5, 164

40 Farrell, P.A. Review of ACTH in exercise Exercise Endocrinology Walter de Grufer \& Co., Berlin, 1985

41 Carli, G. Hormonal and metabolic effects following a football match Int J Sports Med 1986, 7, 36-38

42 Petralgia, F. Response of circulating adrenocorticotrophin, beta-endorphin, beta-lipotropin and cortisol to athletic competition Acta Endocrinol (Copenh) 1988, 118, 332-336

43 Fellmann N. Effect of endurance training on the androgen response to exercise in man Int J Sports Med 1985, 6, 215-219

44 Kinderman, W. Catecholamines, growth hormone, cortisol, insulin and sex hormones in anaerobic and aerobic exercise Eur J Appl Physiol 1982, 49, 389-399

45 Aakvaag, A. Hormonal changes in serum in young men during prolonged physical strain Eur J Appl Physiol 1978, 39, 283-291

46 Opstad, K. Altered physical response to short-term bicycle exercise in young men after prolonged physical strain, calorific deficiency, and sleep deprivation Eur J Appl Physiol 1980, 45, 51-62

47 Urhansen, A. Behaviour of testosterone, SHBG, and cortisol before and after a triathlon competition Int J Sports Med 1987, 8 (5), 305-308

48 Harkonnen, M. Biochemical indicators in diagnosis of overstrain condition in athletes Sp Med and Ex Sci Proceedings of the Olympic Scientific Congress, July, Eugene, Oregon USA, 1984

49 Gmunder, F.K. Effect of long term physical exercise on lymphocyte reactivity: similarity to spaceflight reactions Aviation Space and Environmental Medicine 1988, 59, 146-151

50 Burke, L.S. and Tan, S.A. The suppressive effect of stress from acute exhaustive exercise on T lymphocyte helper/suppressor cell ratio in athletes and non-athletes (Abstract) Med Sci Sports Exerc 1985, 17, 706

51 Cameron, A. and Keast, D. 'Haematological profiles and polyclonal lymphocyte function in continuous and intermittent exercise' MSc thesis, University of Western Australia, 1987

52 Makinnon, L. The effect of exercise on secretory and natural immunity Adv Exp Med Biol 1987, 216A, 869-876

53 Nash, H.L. Can exercise make us immune to disease? Physician and Sports Med 1986, 14, 250-253

54 Besedovsky $\mathbf{H}$, del Ray, A and Sorkin, E. Immunologicalneuroendocrine feedback circuits. In: Guillemin et al (eds). Neural Modulation of Immunity. Raven Press: New York, 1985

55 Stein, M. Bereavement stress and immunity. In Neural Modulation of Immunity (ed Guillemin et al.), Raven Press, New York, 1985

56 Felig, P. Amino acid metabolism in man Ann Reo Biochem 1975, 44, 993-995

57 Ardawi, S. and Newsholme, E.A. Glutamine metabolism in lymphocytes of the rat Biochem J 1983, 212, 835-842

58 Newsholme, E. Biochemistry for the medical sciences John Wiley, Chichester, UK, 1983

59 Roberts, J.A. Loss of form in young athletes due to viral infection $\mathrm{Br}$ Med J 1985, 290, 357-358 